\title{
Avaliação do desempenho clínico de restaurações ART (Tratamento Restaurador Atraumático)
}

\section{Clinical performance Evaluation of Restorations ART (Atraumatic Restorative Treatment)}

\author{
Juliana Pazuch* \\ Marcelo Zottis* \\ Berenice Perussolo ${ }^{* * *}$ \\ Eduardo Grigollo Patussi**** \\ Larissa Corrêa Brusco Pavinato ${ }^{* * * * *}$ \\ Juliane Bervian ${ }^{* * * * *}$
}

\section{Resumo}

Objetivo: avaliar o desempenho clínico de restaurações realizadas com a técnica do tratamento restaurador atraumático (ART) em crianças atendidas na Clínica de Extramuros Faculdade de Odontologia da Universidade de Passo Fundo na escola Monteiro Lobato no ano de 2011. Materiais e método: Inicialmente foram avaliados os prontuários de todas as crianças atendidas no primeiro e segundo semestre de 2011, em seguida foram selecionadas e rechamadas somente as crianças com registro da realização de restaurações com a técnica do tratamento restaurador atraumático (ART). A necessidade de reparo das restaurações de ART foi avaliada em diferentes intervalos de tempo, 12 e 18 meses, conforme o número de faces restauradas, tipo de dentição, dente, idade e gênero da criança. Para o exame das restaurações, utilizou-se os critérios de avaliação de acordo com a metodologia de Phantumvanit et al. (1996). Para análise estatística, utilizou-se o teste Qui-quadrado e o teste Exato de Fisher. Resultados: A análise evidenciou que não houve associação significativa entre o desfecho do estudo (necessidade de tratamento) e as variáveis independentes analisadas ( $p>$ $0,05)$. Conclusão: $O$ desempenho clínico das restau- rações (ART), após 12 meses foi insatisfatório, pois 15 restaurações $(62,5 \%)$ estavam presentes, mas apresentavam necessidade de reparo. O número de faces envolvidas nas restaurações mostrou-se significativo para a necessidade de reparo, uma vez que quando mais faces estavam envolvidas no tratamento, maior a necessidade de reparo $(p=0,012)$.

Palavras-chave: Tratamento restaurador atraumático. Cimento de ionômero de vidro.

\section{Introdução}

Sabe-se que a cárie dentária é uma doença resultante do desequilíbrio nos processos de desmineralização-remineralização que ocorrem constantemente na cavidade bucal. Há estudos que mostram os sinais da doença, os quais estão representados em uma escala de progressão que vai desde da perda inicial de minerais ao nível subclínico até a destruição total do dente ${ }^{1}$.

\footnotetext{
Cirurgiã dentista, Curso de Odontologia da Faculdade de Odontologia da Universidade de Passo Fundo - UPF, Passo Fundo, RS, Brasil. * Cirurgião dentista, Curso de Odontologia da Faculdade de Odontologia da Universidade de Passo Fundo - UPF, Passo Fundo, RS, Brasil. Mestre, Professora da disciplina de Clínica da Criança e do Adolescente da Faculdade de Odontologia da Universidade de Passo Fundo UPF, Passo Fundo, RS, Brasil.

**** Doutor, Professor da disciplina de Clínica da Criança e do Adolescente da Faculdade de Odontologia da Universidade de Passo Fundo UPF, Passo Fundo, RS, Brasil.

***** Doutora, Professora da disciplina de Clínica da Criança e do Adolescente da Faculdade de Odontologia da Universidade de Passo Fundo - UPF, Passo Fundo, RS, Brasil.

${ }^{* * * * * *}$ Doutora, Professora da disciplina de Clínica da Criança e do Adolescente da Faculdade de Odontologia da Universidade de Passo Fundo - UPF, Passo Fundo, RS, Brasil.
} 
Com o entendimento do processo de desmineralização e remineralização, da progressão da doença e da importância do papel das bactérias e do flúor criou-se um novo conceito para o tratamento das lesões de cárie no sentido de utilizar técnicas restauradoras mais preventivas e menos invasivas ${ }^{2}$.

Surge, então, na Tanzânia, uma região de baixo poder aquisitivo e pouco acesso a tratamento dental, a técnica de Tratamento Restaurador Atraumático (TRA), implementada para atender populações africanas sem acesso aos tratamentos odontológicos tradicionais ${ }^{3}$. O tratamento restaurador atraumático consiste basicamente em um método de remoção das lesões de cárie por instrumentos manuais ${ }^{4,5}$, dispensando o uso da eletricidade e de equipamentos odontológicos de custo elevado ${ }^{6}$. Além de ter baixo custo e fácil manuseio, apresenta uma imensa aplicabilidade: pacientes com pouca idade, pacientes fóbicos e pacientes com necessidades especiais ${ }^{7}$. Salienta-se que não há necessidade de anestesia local, já que a técnica é limitada pela sensibilidade que o paciente relata, reduzindo o trauma psicológico dos pacientes ${ }^{6}$.

Quanto às indicações, a técnica do ART elege, principalmente, dentes com lesões de cárie oclusal e ocluso-proximal de dentes decíduos e permanentes. Todavia, o ART está contraindicado quando há a presença de abscessos, fístulas, exposição pulpar, ou ainda quando não favoreça 0 acesso à cavidade com instrumentos manuais ${ }^{8,9}$.

O tratamento restaurador atraumático pode ser indicado também em cavidades atípicas, nas quais há grande destruição de tecido dentário ${ }^{10}$. Sendo assim, a paralização do processo carioso por meio dessa técnica evita que cavidades tornem-se mais extensas e doloridas ${ }^{11}$, podendo-se evitar, principalmente, que muitos dentes sejam extraídos de forma indiscriminada ${ }^{7}$.

Os primeiros estudos clínicos com o ART avaliaram apenas a retenção do material restaurador ao longo do tempo. Pesquisas subsequentes introduziram outros critérios de avaliação e o uso de outros materiais a efeito de comparação e/ou controle ${ }^{6}$.

O objetivo deste estudo foi avaliar o desempenho clínico de restaurações realizadas com a técnica do tratamento restaurador atraumático (ART) em crianças atendidas na Clínica de Extramuros Faculdade de Odontologia da Universidade de Passo Fundo na escola Monteiro Lobato no ano de 2011.

\section{Materiais e método}

Este estudo caracterizou-se por ser um estudo transversal.

A população do estudo foi por conveniência composta por todas as crianças atendidas na clínica extramuros da FO-UPF da escola Monteiro Lobato no ano de 2011 que se submeteram a tratamento restaurador com a técnica ART (Tratamento Restaurador Atraumático).

Primeiramente, foram avaliados os prontuários das crianças atendidas no ano de 2011, em seguida foram selecionadas apenas as crianças com registro da realização de restaurações com a técnica do tratamento restaurador atraumático (ART). Essas crianças foram rechamadas para o exame visual das restaurações.

As restaurações foram avaliadas algumas após 12 e outras após 18 meses, conforme o número de faces restauradas e tipo de dentição. Os critérios de avaliação foram realizados de acordo com a metodologia de Phantumvanit et al. ${ }^{12}$ (1996) conforme apresentação na Tabela 1. As restaurações com escores 0,1 e 7 foram consideradas sem necessidade de reparo, enquanto que aquelas com escores $2,3,4$ e 8 foram consideradas com necessidade de reparo. Nos escores 5, 6 e 9 foram excluídas as restaurações da amostra.

Foram digitados os dados no programa Excel e, posteriormente, exportados para o programa SPSS v. 18.0 para análise estatística. Foram descritas as variáveis categóricas por frequências e percentuais. Para avaliar a associação entre variáveis categóricas com mais de duas categorias foi utilizado o teste Qui-quadrado e no caso de variáveis com duas categorias, comparadas entre dois grupos, foi utilizado o teste Exato de Fisher ou teste de Qui-quadrado com correção de Yates. Quando pelo menos uma categoria teve um valor observado diferente do esperado, foi realizada uma análise de resíduos para detectar qual categoria era mais frequente do esperado. Foi considerado um nível de significância de $5 \%$ para as comparações estabelecidas. 
Tabela 1 - Critérios de Avaliação das restaurações com a técnica de ART

\begin{tabular}{l|l}
\hline \multicolumn{1}{c|}{ Código } & \multicolumn{1}{c}{ Situação } \\
\hline 0 & Presente, sem defeitos. \\
1 & Presente, pequenos defeitos na margem e/ou desgaste de menos $0,5 \mathrm{~mm}$ de profundidade, não necessita de reparo. \\
2 & Presente, pequenos defeitos na margem e/ou desgaste de $0,5 \mathrm{~m} 1 \mathrm{~mm}$ de profundidade, necessita de reparo. \\
3 & Presente, defeitos grosseiros na margem e/ou desgaste de $1 \mathrm{~mm}$ ou mais de profundidade, necessita de reparo. \\
4 & Ausente, restauração (quase) completamente perdida, necessitando de retratamento. \\
5 & Ausente, outro tratamento foi realizado por qualquer outro motivo. \\
6 & Dente ausente, devido a qualquer outro motivo. \\
7 & Restauração presente, desgaste inferior a 0,5 mm de profundidade, não necessita de reparo. \\
9 & Avaliação não é possível. \\
\hline Fonte: Phantumo presente, desgaste superior a $0,5 \mathrm{~mm}$ de profundidade, necessita de reparo.
\end{tabular}

Fonte: Phantumvanit ${ }^{12}$ (1996).

\section{Resultados}

Das 59 crianças selecionadas para serem rechamadas para avaliação, com registro de realização de restauração com a técnica do $\mathrm{ART}$, dessas 36 não compareceram ou não conseguiram ser contatadas, as 23 avaliadas contabilizaram 48 restaurações examinadas. Das restaurações examinadas, 24 foram excluídas por apresentarem restaurações categorizadas com escores 5 e 6 (Tab. 1). Assim, após 12 e 18 meses da realização das restaurações, $9(37,5 \%)$ restaurações estavam presentes sem necessidade de reparo e $15(62,5 \%)$ restaurações estavam presentes com necessidade de reparo (Tab. 2).

Tabela 2 - Frequência da variável dependente

\begin{tabular}{l|c|c}
\hline \multirow{2}{*}{ Variável dependente } & \multicolumn{2}{|c}{ Frequência } \\
\cline { 2 - 3 } & $\mathrm{N}$ & $\%$ \\
\hline Sem necessidade de reparo & 9 & 37,5 \\
Com necessidade de reparo & 15 & 62,5 \\
Total & 24 & 100 \\
\hline
\end{tabular}

Observou-se, de acordo com as variáveis independentes, que a frequência de crianças do gênero masculino e feminino não teve significância (Tab. 3). Com relação à idade, constatou-se uma frequência maior de crianças na faixa etária de sete a nove anos (50\%). Quando considerado a dentição, o dente que recebeu a restauração e o número de faces envolvidas, a maioria foi realizada na dentição decídua $(79,17 \%)$, sendo $50 \%$ realizadas em posteriores superiores, com uma face envolvida em $37,5 \%$ das restaurações avaliadas (Tab. 3) e duas faces envolvidas em $29,16 \%$.

Dos dentes avaliados, os anteriores e também aqueles com cinco faces restauradas, nenhum precisou de reparos.
A análise univariada (teste qui-quadrado) evidenciou que não houve associação significativa entre o desfecho do estudo (necessidade de tratamento) e as variáveis independentes idade da criança ( $p$ $<0,202)$, gênero da criança $(p<0,597)$, dentição $(p$ $<0,897)$, dente $(p<0,099)$ e tempo das rechamadas $(p<0,722)$. Sendo significativo somente no número de faces $(p<0,012)$, (Tab. 4).

Tabela 3 - Frequências das variáveis independentes

\begin{tabular}{l|c|c}
\hline \multirow{2}{*}{ Variáveis Independentes } & \multicolumn{2}{|c}{ Frequência } \\
\cline { 2 - 3 } & $\mathrm{N}$ & $\%$ \\
\hline Gênero da criança & 11 & $45,83 \%$ \\
$\quad$ Masculino & 13 & $54,17 \%$ \\
$\quad$ Feminino & & \\
Idade da criança & 8 & $33,33 \%$ \\
5-6 anos & 12 & $50 \%$ \\
$7-9$ anos & 4 & $16,67 \%$ \\
>=10 anos & & \\
Dentição & 19 & $79,17 \%$ \\
Decídua & 5 & $20,83 \%$ \\
Permanente & & \\
Dente & 4 & $16,67 \%$ \\
Anteriores & 8 & $33,33 \%$ \\
Posteriores inferiores & 12 & $50 \%$ \\
Posteriores superiores & & \\
№ de faces & 9 & $37,5 \%$ \\
1 face & 7 & $29,16 \%$ \\
2 faces & 4 & $16,67 \%$ \\
3 faces & 4 & $16,67 \%$ \\
5 faces & 10 & $100 \%$ \\
Tempo & 14 & $58,33 \%$ \\
12 meses & & \\
18 meses & & \\
\hline
\end{tabular}


Tabela 4 - Cruzamento do desfecho necessidade de tratamento com as variáveis independentes

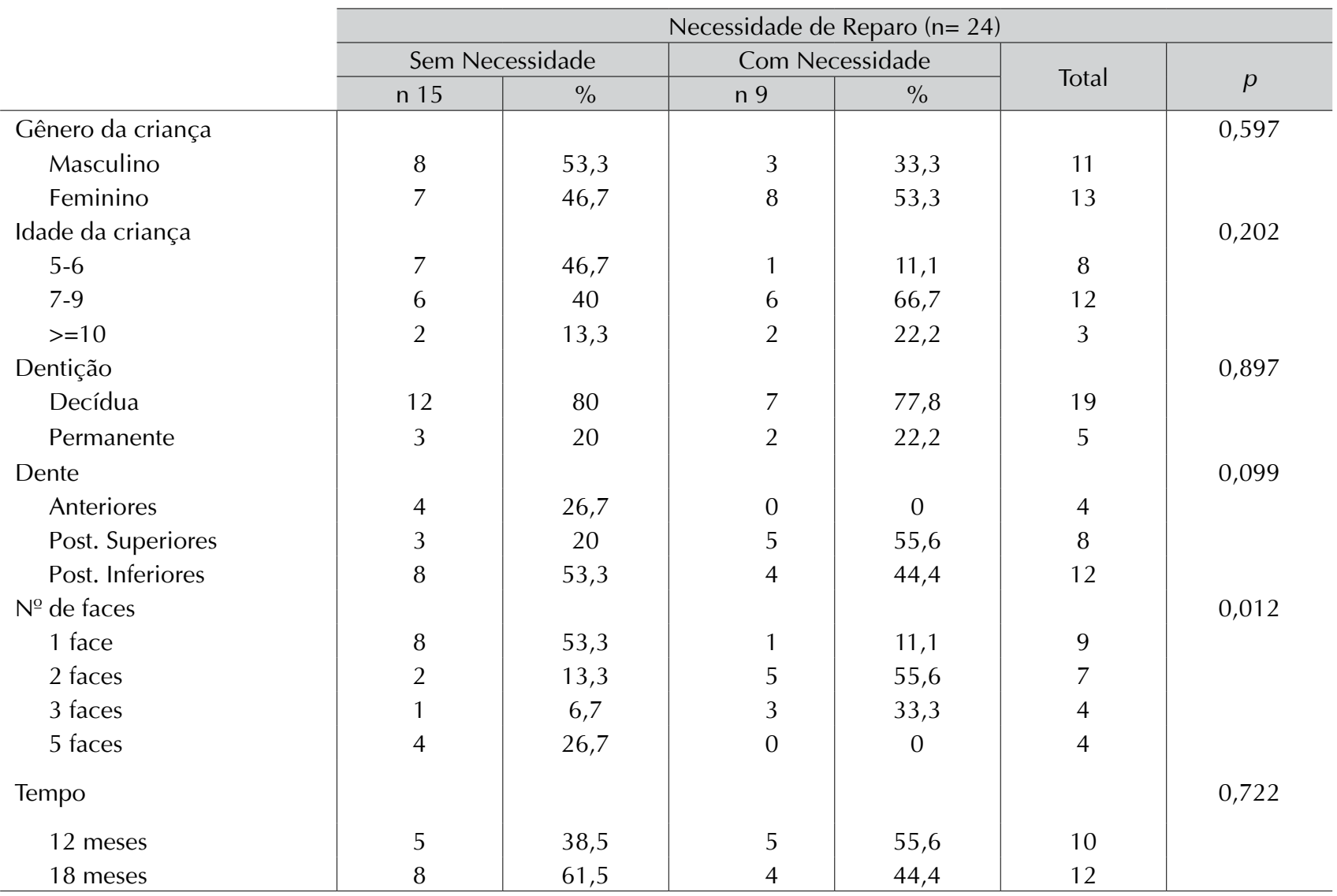

Análise estatística Teste de $\mathrm{P} ; \mathrm{p} \leq 0,05$ é considerado significativo.

\section{Discussão}

Atualmente, há grande preocupação da comunidade odontológica em realizar restaurações que exijam a menor destruição do dente. Com essa filosofia, aliada à facilidade de realização, indica-se o Tratamento Restaurador Atraumático que envolve procedimentos educativos e preventivos, assim como uma parte restauradora ${ }^{2,11,13}$.

Pode-se afirmar que o ART segue o conceito atual de intervenção mínima, no sentido de preservar a estrutura dentária, pois permite uma terapia pouco invasiva ${ }^{5,6,13,14}$, centrada na redução do número de bactérias e no aporte de nutrientes pelo selamento da cavidade. Esse mecanismo considera o grande potencial de reparação da dentina afetada, pela redução do metabolismo bacteriano e favorecimento da resposta do complexo dentino-pulpar, com o auxílio de materiais que favorecem a reorganização dos tecidos dentários e a inibição do crescimento bacteriano $0^{8,11}$.

A técnica do ART caracteriza-se pela remoção de tecido cariado por meio da escavação da dentina infectada com instrumentos manuais, sob isolamento relativo, sem anestesia e, restauração com material adesivo e liberador de flúor, o cimento de ionômero de vidro ${ }^{15}$. É uma forma simplificada de atendimento, que dispensa o uso de anestesia e iso- lamento absoluto. Nos casos referidos neste estudo a técnica foi realizada adequadamente, bem como foram obedecidos os padrões de biossegurança ${ }^{15}$.

Segundo a literatura, o ART é usado para restaurar e prevenir cáries em pacientes jovens, pacientes não colaboradores, ou pacientes com necessidades especiais ou ainda em casos em que não há condições adequadas para o preparo cavitário convencional ${ }^{15}$. Neste estudo, foram avaliadas 59 crianças na idade entre 5 e 10 anos, a variável idade da criança não apresentou associação significativa com a variável dependente do estudo, a necessidade de reparo das restaurações $(p<0,202)$. Provavelmente, se o estudo fosse realizado com uma amostra de crianças de menor idade, a associação seria mais facilmente verificada, como citada por outros autores ${ }^{16,17}$.

Observa-se uma mudança na reação das crianças na realização de tratamento odontológico convencional e em tratamento realizado pela técnica ART, no que diz respeito à ansiedade, principalmente em crianças menores. Na maioria das vezes, durante o tratamento odontológico das crianças tratadas pela técnica restauradora atraumática, os índices de tranquilidade mostram-se significativamente melhores se comparados aos índices alcançados nos atendimentos pela técnica convencional ${ }^{18}$. 
Estudos suportam que as restaurações com ART são altamente efetivas em prevenir lesões de cárie e na manutenção de saúde bucal ${ }^{2,19}$. Para que as restaurações realizadas sejam verdadeiramente efetivas necessita-se de uma manutenção periódica para que questões como dieta e higiene sejam permanentemente reforçadas ${ }^{13}$. Assim, as restaurações de ART são verificadas e a necessidade de reparo demonstra o desempenho clínico da restauração. No grupo estudado, dificilmente a família era membro integrante do tratamento. Como a amostra foi de conveniência e as crianças recebiam tratamento na escola, os pais permitiam o tratamento, mas não auxiliaram no controle dos outros fatores etiológicos da doença em casa. Isso, infelizmente, resulta em tratamento com alto número de insucessos, em que a lesão é tratada, e a doença não ${ }^{14,20}$.

Neste estudo, a análise univariada (teste qui-quadrado) evidenciou não ser possível uma associação significativa entre o desfecho do estudo (necessidade de reparo) e o tempo entre as rechamadas $(p<0,722)$, bem como as outras variáveis associadas. Acredita-se que este estudo sirva como modelo inicial e que uma ampliação no período de acompanhamento e do tamanho da amostra possibilite a visualização de outras associações e significância.

Cada vez mais, estudos vêm testando a eficácia da técnica da ART, inclusive em molares permanentes ${ }^{11} \mathrm{e}$ salienta-se a importância no intuito de minimizar o número de extrações dentárias realizadas nos países subdesenvolvidos ${ }^{21}$.

A utilização do material cimento de ionômero de vidro (CIV) foi eleito como material de escolha para o ART devido às propriedades químicas e físicas estarem apropriadas para uma técnica pouco invasiva. A liberação de fluoretos é o grande fator determinante que influencia a preferência pela escolha desse material para realização das restaurações atraumáticas ${ }^{9}$. O CIV tem comprovado potencial de adesão por meios físico-químicos que apresentam tanto ao esmalte quanto à dentina, possibilitando, dessa forma, considerável economia de tecido dental sadio. Recentemente, foram desenvolvidas novas formulações de CIV convencional para ART em que o tempo de presa é reduzido e a sensibilidade à umidade do material é controlada, resultando em ganho nas propriedades físicas do material ${ }^{22}$. Estudos clínicos randomizados vem sendo acompanhados e comprovam a eficácia do cimento ${ }^{23,24} \mathrm{e}$ a relevância clínica na utilização do material ${ }^{17,25}$.

Dessa forma, verificando os resultados encontrados, apesar da alta necessidade de reparo das restaurações realizadas com a técnica do ART (62,5\%), é importante ressaltar a importância desse tratamento, em função de facilitar a introdução de técnicas restauradoras pelos alunos da graduação, permitindo o estabelecimento de uma nova filosofia minimamente invasiva na Odontologia, controlando a progressão da doença ao mesmo tempo em que trata seus sintomas ${ }^{20,22}$.
Por fim, o baixo desempenho clínico das restaurações leva-nos a acreditar que o isolamento relativo feito pelos alunos junto com a impaciência dos pacientes possam ser fatores que prejudiquem a longevidade clínica dessas restaurações, a falta de experiência com a técnica e/ou manejo com o paciente, além da falta de integração com a família podem ser outros fatores influentes no resultado. No entanto, isso demonstra a importância do conhecimento técnico científico para o acadêmico, pois o mercado de trabalho exige adaptação da técnica, do manejo e demanda excelência no desempenho clínico.

\section{Conclusões}

O desempenho clínico das restaurações (ART), de acordo com os critérios estabelecidos neste estudo foi insatisfatório, pois mais da metade das restaurações presentes, necessitavam de reparos. Observou-se que quanto maior o número de faces envolvidas no procedimento restaurador de um mesmo dente, maior foi a necessidade de reparo.

Diante disso, nossos resultados sugerem mais estudos, de ensaios clínicos randomizados, com maior poder de gerar evidência cientifica, com amostras maiores para que o desempenho clínico da técnica (ART) possa ser indicado com excelência.

\section{Abstract}

Objective: To clinically assess restorations performed with the atraumatic restorative treatment (ART) technique in children treated at the Extramural Clinic School of Dentistry, University of Passo Fundo, in the Monteiro Lobato School in 2011. Materials and method: The medical records of all children assisted in the first and second semester of 2011 were initially assessed, then only children subjected to the atraumatic restorative treatment (ART) technique were selected and called back. The need for repair of ART restorations was assessed at different time intervals of 12 and 18 months, considering the number of surfaces restored, type of dentition, tooth, age, and gender of the child. The assessment criteria according to the methodology by Phantumvanit et al. (1996) were used for examining the restorations. The chi-square test and Fisher's exact test were used for statistical analysis. Results: The analysis showed no significant association between the study outcome (need for treatment) and independent variables ( $p>0.05$ ) analyzed. Conclusion: The clinical performance of restorations (ART) after 12 months was unsatisfactory, because 15 restorations (62.5\%) were present but needed repair. The number of surfaces involved in restorations was significant to the need for repair, considering the more surfaces involved in the treatment, the greater the need for repair $(p=0.012)$.

Keywords: Atraumatic restorative treatment. Glass ionomer cement. 


\section{Referências}

1. Maltz M, Alves LS. Pulp capping material is an important prognostic factor for direct pulp capping in permanent teeth. J Evid Based Dent Pract 2013; 13(3): 117-9.

2. Frencken JE, Peters MC, Manton DJ, Leal SC, Gordan VV, Eden E. Minimal intervention dentistry for managing dental caries - a review. Int Dent J 2012; 62(5):223-43.

3. Frencken JE, Holmgren CJ. Tratamento Restaurador Atraumático (ART) para a Cárie Dentária. São Paulo: Santos; 2001.

4. Tascon J. Restauración atraumática para el control de la caries dental: historia, características y aportes de la técnica. Rev Panam Salud Publica 2005; 17(2):110-5

5. Frencken JE, Makoni F, Sithole WD. ART restorations and glass ionomer sealants in Zimbabwe: survival after 3 years. Com Dent Oral Epidemiol 1998; 26(6):372-81.

6. Frencken JE, Songpaisan Y, Phantumvanit P, Pilot T. An atraumatic restrorative treatment (ART) technique: evaluation after one year. In Dent J 1994; 44(5):460-4.

7. Raggio DP, Politano GT, Imparato JCP. Tratamento Restaurador Atraumático (ART): Filosofia e técnica de trabalho. In: Bussadori SK. e cols. Remoção química e mecânica do tecido cariado: abordagem sobre o tratamento minimanete invasivo da doença cárie. São Paulo: Santos; 2010. cap. 11, 133-9.

8. Massoni AC de LT, Pessoa CP, Oliveira AFB de. Tratamento Restaurador Atraumático e suas aplicações na Saúde Pública. Rev Odontol UNESP 2006; 35(3):201-7.

9. Imparato JCP, Delmondes FS, Rodrigues-Filho L. Selamento provisório de primeiros molares permanentes em erupção. In: Imparato JCP. Tratamento Restaurador Atraumático. Técnicas de mínima intervenção para o tratamento da doença cárie dentária. Curitiba: Editora Maio Cap. 9, p. $135-47 ; 2005$

10. Carvalho LS, Aldrigui JM, Bonifácio CC, Imparato JCP, Raggio DP. Tratamento Restaurador Atraumático em cavidades atípicas. Rev Gaúcha Odontol 2009; 57(3):357-62.

11. Maltz M, Alves LS. Incomplete caries removal significantly reduces the risk of pulp exposure and post-operative pulpal symptoms. J Evid Based Dent Pract 2013; 13(3):120-2.

12. Phantumvanit $P$. atraumatic restorative treatment technique (ART): a three-year community Field Trial in Thailand - survival o fone - surface restorations in the permanente dentition. J Public Health 1996; 56(3):141-5.

13. Kateeb ET, Warren JJ, Damiano P, Momany E, Kanellis M, Weber-Gasparoni $\mathrm{K}$, et al.Teaching atraumatic restorative treatment in U.S. dental schools: a survey of predoctoral pediatric dentistry program directors. J Dent Educ 2013; 77(10):1306-14

14. Luengas-Quintero E, Frencken JE, Muñúzuri-Hernández JA, Mulder J. The atraumatic restorative treatment (ART) strategy in Mexico: two-years follow up of ART sealants and restorations. BMC Oral Health 2013; 8:13:42.

15. Organização Mundial da Saúde. Guia prático para a técnica do tratamento restaurador atraumático, Groningen, p. 47; 1993.

16. Lee GH, McGrath C, Yiu CK. The care of the primary dentition by general dental practitioners and paediatric dentists. Int Dent J 2013; 63(5):273-80.
17. Bonifácio CC, Hesse D, de Oliveira Rocha R, Bönecker M, Raggio DP, van Amerongen WE. Survival rate of approximal-ART restorations using a two-layer technique for glass ionomer insertion. Clin Oral Investig 2013; 17(7):1745-50.

18. Schriks MCM, Van Amerongen WE. Atraumatic perspective of ART: psychological aspect of treatment with or without rotary instruments. Com Dent Oral Epidemiol 2003; 31(1):15-20

19. Cefaly DF, Tapety CM, Mondelli RF, Lauris JR, Phantumvanit P, Navarro MF. Three-year evaluation of the ART approach in Class III and V restorations in permanent anterior teeth. Caries Res 2006; 40(5):389-92.

20. Figueiredo MC. Research proposal: quantitative and qualitative evaluation of the ART in infants. J Appl Oral Sci 2006; 14 (special Issue):20-4.

21. Lima DC de, Saliba N, Moimaz SAS. Tratamento restaurador atraumático e sua utilização em saúde publica. Rev Gaúcha Odontol 2008; 56(1):75-9.

22. Figueiredo KSP de, Forte FDS, Sampaio FC. Desempenho clínico de restaurações ART (Tratamento Restaurador Atraumático) em crianças atendidas na clínica de cariologia da UFPB. Rev Odontol UNESP 2008; 37(4):351-5.

23. Gil-Montoya JA, Mateos-Palacios R, Bravo M, González-Moles MA, Pulgar R. Atraumatic restorative treatment and Carisolv use for root caries in the elderly: 2-year follow-up randomized clinical trial. Clin Oral Invest 2014; 18(4):1089-95.

24. Holmgren CJ, Lo EC, Hu D. Glass ionomer ART sealants in Chinese school children-6-year results. J Dent 2013; 41(9):764-70.

25. Mijan M, de Amorim RG, Leal SC, Mulder J, Oliveira L, Creugers NH, et al. The 3.5-year survival rates of primary molars treated according to three treatment protocols: a controlled clinical trial. Clin Oral Invest 2013; 90(3):347-53

Endereço para correspondência:

Juliane Bervian

Faculdade de Odontologia da

Universidade de Passo Fundo - UPF

99052-900 Passo Fundo/RS

Fone: (54) 3316-8402

E-mail: jbervian@upf.br

Recebido: 05/12/2013. Aceito: 02/04/2014. 